\title{
IIIness related deception: social or psychiatric problem?
}

\author{
Christopher Bass ${ }^{1} \quad$ Peter W Halligan²
}

J R Soc Med 2007;100:81-84

\section{SUMMARY}

In this paper we question the validity of factitious disorder as a meaningful psychiatric diagnosis. When the diagnosis is used there is often the assumption that the person engaging in the 'deception' is not lying in the traditional sense of being deliberately misleading. Moreover, little is known about the aetiology or psychopathology underlying factitious disorder, and the legitimacy of deception as a mental disorder has been questioned. It is argued that while illness deception may be more common that hitherto assumed, factitious disorder as a distinct type of psychiatric disorder is conceptually flawed, diagnostically impractical and clinically unhelpful and should be dropped from existing nosologies.

\section{INTRODUCTION}

Deception (including lying) - the deliberate distortion of information intended to mislead others - is a ubiquitous form of social behaviour that all people, at some time, engage in. ${ }^{1,2}$ Indeed, there is growing evidence from evolutionary studies, normal human child development and developmental psychopathology to suggest that deception is an ability that develops naturally during childhood. ${ }^{3}$ The broadsheet newspapers regularly report articles about financial, benefit, prescription, credit card, property and insurance fraud, ${ }^{4}$ but everyday forms of deception are more difficult to establish, presumably because they are more successful. Generally, people are good at lying but rather poor at detecting lies or liars. This failure to detect deception may be explained by the fact that many lies go undetected and because the observer is not motivated or pre-disposed to consider the possibility. ${ }^{1}$ According to Vrij, most of us on average lie about twice a day or in one quarter of interactions with others, and hence deception as the deliberate communication of information believed to be false is an essential part of negotiating every day social interaction. ${ }^{1,5}$ A recent survey of National Institutes of Health-funded scientists found that a third of respondents admitted to engaging in a variety of deceptive practices including fabrication, falsification, or plagiarism in their research. ${ }^{6}$

'Department of Psychological Medicine, John Radcliffe Hospital, Oxford OX3 9DU, UK ${ }^{2}$ School of Psychology, Cardiff University, UK

E-mail: christopher.bass@obmh-tr.nhs.uk
In this paper we suggest (i) that existing psychiatric accounts of illness deception underestimate the contribution from non-medical deception; (ii) the continued specialized psychiatric use of the term deception in such cases should be dropped, given that if the behaviour is involuntary and largely outside the subjects conscious control then it does not constitute deception as the term is normally used; and (iii) that at a more pragmatic clinical level it is not clear how current psychiatric accounts can meaningfully and reliably distinguish the specialist type of deception attributed to factitious disorders from the non-medical accounts. ${ }^{7}$

\section{PREVALENCE OF NON-MEDICAL ILLNESS DECEPTION}

Given the potential benefits attached to the sick role, all patients (except those with established diminished capacity due to brain damage or psychiatric illness) have the potential and opportunity to exaggerate symptoms for reasons other than but including the benefits of the sick role. This is not to say that many actually engage in such behaviours but (in the absence of a reliable evidence base) it would be wise to assume that illness related deception is probably no less common than other forms of deception found in society.

Central to this account of illness deception are choice ${ }^{8}$ and personal responsibility - the building blocks for morality, democracy, and justice. Without the explicit assumption of volitional choice, any discussion of illness deception is rendered incoherent. Where a subject's capacity to exercise choice or control (including insight) is compromised by brain damage or psychiatric disorder then it not meaningful to attribute deception (as commonly used) and as such the term is best avoided in clinical practice.

Because deception is commonly found in many domains of human behaviour, there is no reason to assume that it is any less common in medical consultations or illness presentations, given not only the personal, social and financial benefits of the sick role but also the relatively low risk of detection. ${ }^{7} \mathrm{~A}$ recent study of 131 practising members of the American Board of Clinical Neuropsychology provided estimates of the prevalence of malingering and symptom exaggeration for a variety of different clinical conditions. ${ }^{9}$ In this study, estimates of the base rate of 
malingering/symptom exaggeration were calculated using over 33,000 annual cases seen by a group of clinical neuropsychologists. The reported base rates (when statistically adjusted to account for the influence of referral source) were $29 \%$ for personal injury, 30\% in the case of disability or workers compensation, $19 \%$ in criminal cases and $8 \%$ in medical or psychiatric cases. The same rates broken down by diagnosis revealed 39\% in the case of mild head injury, 35\% in fibromyalgia and chronic fatigue, 31\% in chronic pain, $15 \%$ in depressive disorders, and $11 \%$ in dissociative disorders. In a separate review of 1363 compensation-seeking cases, Larrabee ${ }^{10}$ found similar figures for mild head injury of $40 \%$.

From a non-medical perspective, illness deception (exaggeration, dissimulation or feigning) can be considered one of several rational / economic /adaptive options open to patients when seeking limited health care and/or social and welfare resources. Moreover, deception is not confined to patients: Wynia et al. ${ }^{11}$ found that $37 \%$ of physicians had 'sometimes, often or very often' been asked to deceive health care payers by their patients; furthermore, $10 \%$ of 700 physicians in the US admitted to fabricating signs or symptoms on behalf of patients and 54\% admitted to deception of insurance payers. In another study of over 400 American physicians, Novack et al. ${ }^{12}$ found that over $80 \%$ were prepared to lie in the interest of their patients. The CBI estimates that one third of all sick notes are bogus and that General Practitioners contribute to illness behaviour. In the UK, a study by Norwich Union Healthcare found that doctors issued 22 million sick notes per year, of which 9 million $(41 \%)$ were thought to be questionable. ${ }^{13}$

\section{IS DECEPTION A VALID MEDICAL CONSTRUCT?}

Viewed as a medical / psychiatric disorder (i.e. a recognized mental condition invoking some form of non-specific unconscious psychopathological processes), illness deception, typically characterized as factitious disorder, is conceptually distinct from everyday usage. The justification for maintaining this type of 'psychiatric' deception (rarely diagnosed outside medico-legal settings) from what we describe as 'non-medical' illness deception hinges largely on the nature of the motivation - the assumed 'psychological need' to adopt the sick role- - together with the assumption (common to many psychiatric conditions) that the behaviour is involuntary and largely outside the subject's conscious control.

Non-medical illness deception however is characterized as qualitatively distinct from psychiatric accounts of illness deception precisely in not being an illness. ${ }^{7}$ Non-medical illness deception is typically understood as a conscious voluntary act or set of actions where the deliberate benefits and/or lack of responsibilities that society and the legal system have bestowed upon the sick role. The critical conceptual difference is not, however, the nature, extent or use of deception per se, but crucially the nature of the motivation that provides for the deception which in turn exculpates the subject from normal responsibility. ${ }^{14}$

Given their underlying 'psychological need', patients with factitious disorder are considered to knowingly fake symptoms (i.e. plan or intend to deceive) for the purpose of adopting the sick role. However, it is possible that the same subject could also engage in deception for reasons of personal, monetary or instrumental benefit which are not mutually exclusive with and indeed may be compatible with seeking the sick role. Thus, the clinical reality of arriving at a reliable and compelling diagnosis of medical deception is complicated by the possibility that several different motivations underlie the desired goal. While the sick role may well be one of the intended goals, it is not possible to ascertain with any degree of reliability that this is the only goal (conscious or unconscious) nor that the targeting of the sick role is without consideration of some of the associated positive personal or 'positive self-presentational outcomes.' ${ }^{2}$ The likelihood that more than one type of conscious intention or motivation could be concurrently involved in most forms of illness behaviour makes this distinction impractical and unrealistic for both diagnosis and legal presentation

\section{PROBLEMS WITH CLINICAL DIAGNOSIS}

Although factitious disorder, with its selective usage of deception, is widely accepted in both ICD-10 and DSM$\mathrm{V},{ }^{14,16}$ the condition is conceptually flawed since it does not provide guidance 'for determining consciousness of actions (voluntariness) or consciousness of motivation' 17 and little by way of evidence for clinicians to differentiate meaningfully between 'non clinical' (i.e. social or non-medical based illness deception) and factitious or fabricated illnesses. ${ }^{18}$ In practical terms, it remains unclear whether it is possible to perform (within the confines of a clinical interview) the seemingly impossible task of inferring the level of conscious awareness, the degree of consciously mediated intention and the motivating goals that accompany (particularly subjective or symptom based) reports of illness). Can clinicians be reasonably expected to distinguish between someone who is motivated to adopt the sick role for reasons more than and/or restricted to the sick role?

Equally problematic is the use of the term deception in these cases. Everyday usage of the term deception describes the attempt to deliberately and wilfully convince someone of events or actions that one knows not to be true. As Spence et al. ${ }^{19}$ point out, this involves several high cognitive processes process (including theory of mind and deontic 
Conceptualising Illness Behaviour

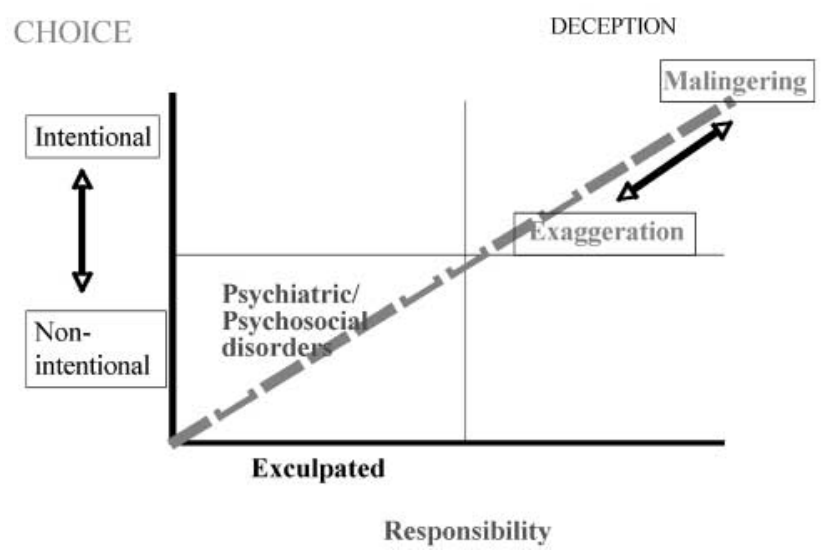

Figure 1 Model of illness deception incorporating patient choice, free will and intentionality

reasoning). If a subject can be shown to be suffering from a recognized diminished capacity (cognitive or psychiatric) where he/she cannot be reasonably expected to exercise choice over their internal motivation or actions, then it seems both unfair and inconsistent to characterise such a person as engaging in deception as typically understood.

Despite declarations that illness deception per se (other than factitious disorders) does not constitute a formal 'mental disorder', in practice most forms of illness deception (malingering included) continue to be implicitly medicalized by clinical researchers ${ }^{20-23}$ within a 'pathogenic model', ${ }^{24}$ which considers all forms of deception to be the product of some unspecified mental disorder. ${ }^{25}$ In the light of this 'diagnostic creep', it is timely to review and where necessary confront the conceptual blurring and diagnostic 'over reach' 26 that continues to impede meaningful discussion of illnesses behaviours involving deception. To this purpose, we propose a simple schematic model for conceptualizing illness deception that involves both psychopathology and subjective choice (Figure 1).

Considered as an act of wilful deception, illness deception can be meaningfully conceptualized within a socio-legal or moral model of human nature that recognizes the capacity for choice and the potential for pursuing, benefits associated with the sick role. This pervasive and deep-seated notion of choice and individual responsibility provides a reasonable framework from which to explain and discuss illness behaviour not produced by disease, injury, psychopathology or psychosocial factors. The emphasis on the non-medical aspects of illness behaviours helps move the discussion away from the traditional reliance on medical 'causes' and encourages a more empirical investigation of the 'reasons', psychosocial factors and potential incentives that explain why individuals might choose to engage in social deviant behaviours.

\section{CONCLUSIONS}

As the conceptual justification for factitious disorders is 'empirically unsubstantiated' and the motivation for diagnostic purposes (conscious versus unconscious; voluntary versus involuntary) essentially unknowable, it seems reasonable to question the clinical status and diagnostic legitimacy of factitious disorder ${ }^{27}$ as a selective medical disorder. Far from clarifying this distinction with nonmedical illness deception, factitious disorders have (if anything) complicated matters further by closing the gap between an unprovable underlying (but as yet unspecified) psychopathological process and a morally questionable but volitional-based choice to deceive others by feigning illness.

Moreover, given the difficulty in establishing a meaningful and reliable diagnosis based on motivation (and not deception), patients whose behaviour and presentation warrant psychiatric help should not have to endure the additional stigma of having their behaviour questioned in terms of motivations other than the sick role and causes other than their illness. In such circumstances, it seems sensible to abandon the traditional 'medically specialized' use of deception and confine its standard non-psychiatric use for subjects who have the capacity (or at least the perceived capacity) to exercise choice and decision making. ${ }^{19}$

\section{REFERENCES}

1 Vrij A. Detecting Lies and Deceit. Wiley: Chichester, 2000

2 Grubin D. Getting at the truth about pathological lying. J Am Acad Psychiatry Law 2005;33:350-3

3 Lewis M, Saarni C. Lying and Deception in Everyday Life. Guildford Press, New York, 1993

4 Cavendish C. Liars, cheats? Join the club. Times 20 October 2005: 24

5 Ford CV. Lies! Lies! Lies! The Psychology of Deceit. Washington, DC: American Psychiatric Press, 1996

6 Martinson BC, Anderson MS, de Vries R. Scientists behaving badly. Nature 2005;435:737-8

7 Halligan P, Bass C, Oakley D. Illness behaviour as wilful deception. In: Halligan P, Bass C, Oakley D (eds). Malingering and Illness Deception. Oxford: Oxford University Press, 2003

8 Henderson S. The neglect of volition. Br J Psychiatry 2005;186:273-4

9 Mittenberg W, Patton C, Vanyock E, Condit D. Base rates of malingering and symptom exaggeration. J Clin Exp Neuropsychol 2002;24:1094-102

10 Larrabee G. Detection of malingering using atypical performance patterns on standard neuropsychological tests. Clin Neuropsychol 2003; 17:410-25

11 Wynia M, Cummins D, Van Geest J, Wilson I. Physician manipulation of reimbursement rules for patients: between a rock and a hard place. JAMA 2000;283:1858-65

12 Novack DH, Detering BS, Farrow L, et al. Physician's attitudes toward deception to resolve difficult ethical problems. JAMA 1989;261:2980-5

13 Lister S. Bogus sick notes give time off to $9 \mathrm{~m}$ a year. Times 28 April 2004

14 Diagnostic and Statistical Manual of Mental Disorders, 5th edition. Text Revision. American Psychiatric Association, 2000 
15 Hamiliton JH Deemer HN, Janata JW. Feeling bad but looking good : Sick role features that lead to favorable interpersonal judgments. $J$ Soc Clin Psychol 2003;22:253-74

16 World Health Organization. Manual of the International Statistical Classification of Diseases and Related Health Problems. $10^{\text {th }}$ edition. Geneva: WHO, 2000

17 Ensalada LH. The importance of illness behaviour in disability management. Occupational Med 2000;15:739-54

18 Turner M. Factitious disorders. Reformulating the DSM-IV criteria. Psychosomatics 2006;47:23-32

19 Spence SA, Hunter M, Farrow T, et al. A cognitive neurobiological account of deception:evidence from functional neuroimaging. Phil Trans R Soc Lond 2004;359:1755-62

20 Miller L, Cartlidge N. Simulation and malingering after injuries to the brain and spinal cord. Lancet 1972;7750:580-5
21 Gorman WF. Neurological malingering. Behavioural Sciences and the Law 1984;2:67-73

22 Hirsch HL. Malingering. Trauma 1998;39:53-88

23 Reynolds CR. Detection of Malingering During Head Injury Litigation. New York: Plenum,1998

24 Rogers R. Introduction. In: Clinical Assessment of Malingering and Deception, $2^{\text {nd }}$ edition. New York: Guidford Press, 1997: 1-19

25 Bordini Ej, Chaknis MM, Ekman-Turner RM, Perna RB. Advances and issues in the differential diagnosis of malingering versus brain injury. Neurorehabilitation 2002; 17:93-104

26 Farah MJ. Emerging ethical issues in neuroscience. Nature Neuroscience 2002;5:1123-9

27 Rogers R, Bagby R, Rector M. Diagnostic legitimacy of factitious disorder with psychological symptoms. Am J Psychiatry 1989;146:1312-4 